\section{Dye transfer: all washed up}

Peroxidases can inactivate the colored dyes that leach out of clothing during the wash, and variants able to withstand the harsh environment of the washing machine are sought by the detergent industry. Directed evolution is a powerful means of altering enzymes to perform under such non-physiological conditions, and in this issue, Cherry et al. use this technology to evolve a highly stable peroxidase from inkcap mushrooms that is ideally suited for laundry detergent applications (see pp. 333 and 379).

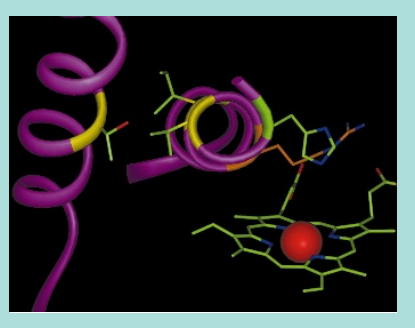

\section{Mirror, mirror}

Chirality is an inherent property of carbonbased compounds, and the ability to discriminate between the enantiomers of a biological molecule is important for applications in archeology, pharmaceutics and the food industry. Until now, techniques to detect enantiomeric impurities have relied on chemical properties of the molecules and have suffered from a low sensitivity. In this issue, Green and colleagues provide a novel antibody-based immunosensor with a 10 fold greater sensitivity to enatiomeric impurities than current sensor methodologies (see p. 371).

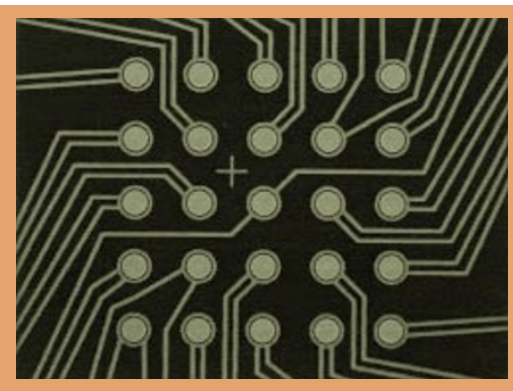

Single nucleotide polymorphisms, or SNPs, are the most prevalent form of genetic variation, and have been found to be associated with a number of human diseases. Efficient strategies to screen genomic DNA for specific mutations are essential for the burgeoning efforts to track SNPs in large populations. Using semiconductor microchips as their platform, Gilles and colleagues have developed a rapid and sensitive method for the discrimination of a complex quadraallelic locus in a gene linked to immunosuppression in pediatric patients (see p. 365).

\section{Knocking out the herpesvirus genome}

Although the herpesvirus genome has been completely sequenced, the roles of many gene products remain unknown. Unlocking these secrets demands strategies to mutate individual genes and see how this affects viral life cycle. To make this easier, Brune et al. have developed a rapid mutagenesis strategy to insert transposons randomly into the herpesvirus genome cloned in bacterial artificial chromosomes (BACs) (see pp. 332 and 360). This method, which should be applicable to any cloned virus, can be used to distinguish rapidly between genes that are essential or nonessential for viral replication.

This month in Nature Biotechnology written by Natalie Dewitt and Robert Frederickson. both tumor growth and metastatic spread in vivo. therapy has been tempered recently due to difficulties in efficiently producing fully active recombinant proteins in suffinow report a promising alternative: the direct gene encoding endostatin into muscle tissue obviating the problems inherent to production of recombinant protein in microorganisms (see pp. 336 and 343$)$.

Corti et al. make safer a promising approach for gene therapy of Parkinson's disease, by creating a system to tightly control expression of the therapeutic Gene delivery techniques have been developed that can genetically modify human neural progenitor cells, proliferate them in culture, and implant them into the CNS. Their twist was to design an adenovirus vector con-

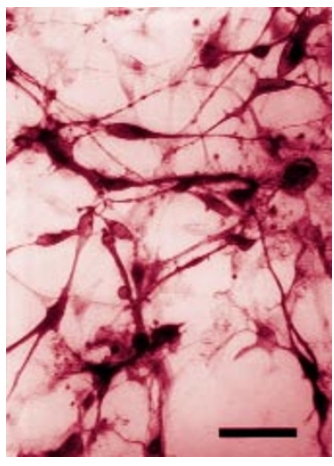

taining an antibiotic-responsive regulatory system that could modulate expression of the therapeutic gene-in this case, the rate-limiting enzyme in L-dopamine synthesis. After the neural cells infected with the vector were transplanted into rats, expression of the gene could be reversibly turned off by injections of the antibiotic.

\section{Gene flow from crops to weeds}

In the ongoing debate about risks of transgenes escape to wild plant populations, data often seem as scarce as opinions are plentiful. Scott and Wilkinson take a critical look at whether transformation of the chloroplast genome can really reduce the risk of transgene escape (see pp. 330 and 390). To test this, they used chloroplast genes as markers to track gene flow from chloroplasts of oilseed rape (a common crop plant) to wild Brassica rapa (a related weed).

Angiostatin and endostatin are proteins that inhibit cient quantities. gene (see p. 349).

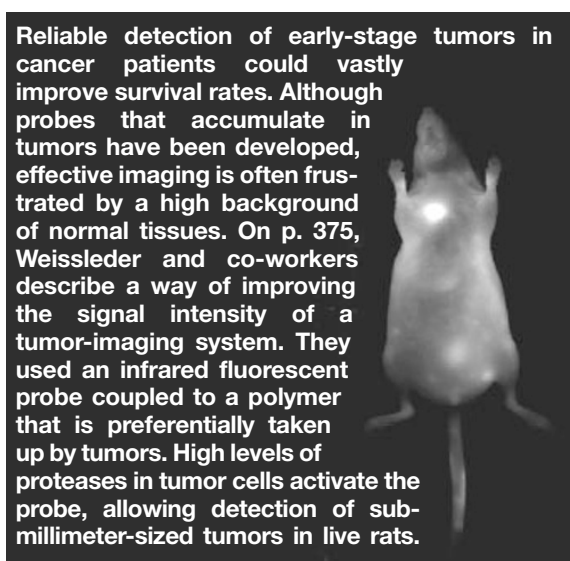

\section{Producing collagen in a mammary bioreactor}

Because of its unusual tensility, collagen has made its way into products as diverse as desserts, shampoo, and bioengineered skin. Usually collagen is extracted from cow hooves and hide, but for medical applications, sources other than rendering lots are needed to ensure the supply is highly purified, active, and pathogen-free. Bulleid and coworkers have turned to mouse mammary glands to produce a recombinant form of procollagen in mouse milk (see p. 385). By co-expressing a collagenhydroxylating enzyme, they produced the mature thermostable form of the protein.

\section{Getting the bugs out}

Escherichia coli has been an indispensable system for cloning recombinant DNA molecules to study gene function and regulation. This workhorse of the molecular laboratory could soon be put out to pasture, however. In this issue, Sykes and Johnston report that PCR products encoding promoters, coding regions, and terminators can be hybridized together and transferred directly into an organism without ligation (see pp. 329 and 355). Surprisingly, these chimeric fragments effect levels of gene expression similar to those observed with genes encoded on plasmids. 